\title{
PERITONITE FECAL POR APENDICITE DE COTO
}

\section{FECAL PERITONITIS CAUSED BY STUMP APPENDICITIS}

Juliana Sopchaki Fagundes ${ }^{1}$; Caroline Teixeira Marques ${ }^{1}$; Jamillah Racki Abu-Ali ${ }^{1}$; Jackson Vinícius de Lima Bertuol2; Thiago André Adame³; Allan Cezar Faria Araujo4.

\section{RESUMO}

Introdução: A apendicite de coto é uma das raras complicações tardias após a apendicectomia, e que pode resultar em apendicite gangrenosa, flegmão, fistula cutânea, perfuração (principal complicação), abscesso intraabdominal e sepse, se não identificada e adequadamente tratada. Caracteriza-se pela inflamação do tecido residual do apêndice pós apendicectomia, com clínica e exames de imagem semelhantes aos da apendicite. O diagnóstico é muitas vezes retardado devido ao baixo índice de suspeita, sendo importante a sua inclusão no diagnóstico diferencial da dor abdominal em quadrante inferior direito pós-apendicectomia. Relato do caso: Relatamos um caso de paciente que apresentou apendicite de coto apendicular 14 anos após apendicectomia, evoluindo para peritonite fecal, com necessidade de peritoniostomia e múltiplas abordagens cirúrgicas. Conclusão: Ainda pouco conhecida e pouco lembrada pelos médicos, a apendicite de coto como hipótese diagnóstica em pacientes com apendicectomia prévia e que apresentam suspeita de abdome agudo e sintoma de dor em FID não deve ser prontamente excluída, uma vez que, apesar de rara, pode levar a complicações graves.

Palavras-chave: Peritonite. Apendicite. Abdome Agudo.

\section{ABSTRACT}

Introduction: Stump appendicitis is one of the rare complications that can be developed after an appendectomy, which can result in gangrenous appendicitis, phlegmon, cutaneous fistula, perforation (main complication), intraabdominal abscess and sepsis, if not identified and properly treated. It is defined as inflammation of the remaining tissue of the appendix after an appendectomy, with symptoms and imaging similar to appendicitis. Diagnosis is often delayed once it is not primarily suspected. Therefore, its rather important to be considered in lower quadrant abdominal pain differential diagnosis. Case report: We report a patients case who presented stump appendicitis 14 years after appendectomy, which evolved into fecal peritonitis, peritoneostomy and demanded several surgical approaches. Conclusion: Still little known and little remembered by physicians, stump appendicitis as a diagnostic hypothesis in patients with previous appendectomy and who present suspected acute abdomen and symptoms of pain in RIF should not be promptly excluded, since despite being rare, can lead to serious complications.

Keywords: Peritonitis. Appendicitis. Abdomen, Acute.

\section{INTRODUÇÃO}

A apendicectomia é um dos procedimentos cirúrgicos mais realizados na prática cirúrgica, e somente no sistema público de saúde brasileiro são realizadas cerca de 100 mil apendicectomias por ano, sendo a maioria por via laparotômica.

Contudo, houve recentemente um grande aumento das apendicectomias por via laparoscópica ${ }^{1}$. Embora o manejo da apendicite aguda somente com antibióticos tenha sido fruto de múltiplos estudos nos últimos anos, o tratamento padrão continua sendo a apendicectomia, cujas complicações incluem infecção de sítio cirúrgico, abscesso intra-abdominal, sangramento, aderências, hérnia incisional e, raramente, apendicite de coto apendicular ${ }^{2}$.

\footnotetext{
${ }^{1}$ Hospital Universitário do Oeste do Paraná - HUOP - Curso de Medicina - Cascavel - PR - Brasil

2Universidade Estadual de Campinas (UNICAMP) - Residência de Cirurgia do Trauma - Campinas - SP - Brasil

${ }^{3}$ Hospital Universitário do Oeste do Paraná - HUOP - Radiologia - Cascavel - PR - Brasil

${ }^{4}$ Hospital Universitário do Oeste do Paraná - HUOP - Cirurgia Geral e Aparelho Digestivo - Cascavel - PR - Brasil
} 
A apendicite de coto é definida pela inflamação e infecção do tecido apendicular remanescente após apendicectomia prévia, o que configura um verdadeiro desafio para o cirurgião. Grande parte dos médicos envolvidos no atendimento inicial de pacientes em pronto-socorro não estão familiarizados com tal patologia, deixando de incluí-la na gama de diagnósticos diferenciais de dor abdominal em quadrante inferior direito (QID) em pacientes já apendicectomizados, tornandose um dilema cirúrgico, uma vez que o diagnóstico e, portanto, o tratamento, acabam sendo tardios, aumentando a morbimortalidade ${ }^{2}$.

Este artigo tem como objetivo o relato de caso de um paciente que apresentou apendicite de coto apendicular 14 anos após apendicectomia, evoluindo para peritonite fecal, necessitando de peritoniostomia e múltiplas cirurgias.

\section{RELATO DO CASO}

Paciente do sexo masculino, 28 anos, deu entrada em serviço terciário de cirurgia com quadro de dor abdominal em quadrante inferior direito (QID) com 25 dias de evolução, a qual progrediu para todo o abdome, associada à distensão abdominal, náuseas, vômitos, diarreia, anorexia e febre. Paciente não apresentava comorbidades ou alergias e possuía apendicectomia por laparotomia mediana infra umbilical realizada há 14 anos.

Ao exame físico estava em regular estado geral, desidratado e taquicárdico. Apresentava abdome distendido, com hiperemia e crepitações em fossa ilíaca direita (FID) e em flanco direito, doloroso difusamente à palpação com sinais de peritonite e plastrão palpável em FID.

Além de exames laboratoriais, apresentava leucocitose, elevação de proteína $\mathrm{C}$ reativa e ausência de disfunções orgânicas. Foi realizada tomografia computadorizada (TC) de abdome com contraste endovenoso.

$\mathrm{Na}$ TC de abdome, conforme evidenciado nas Figuras 1 e 2, foi possivel observar solução de continuidade em região cecal, associada à extensa coleção heterogênea com focos gasosos, que se estendia para subcutâneo do QID e moderada quantidade de líquido livre intraabdominal.

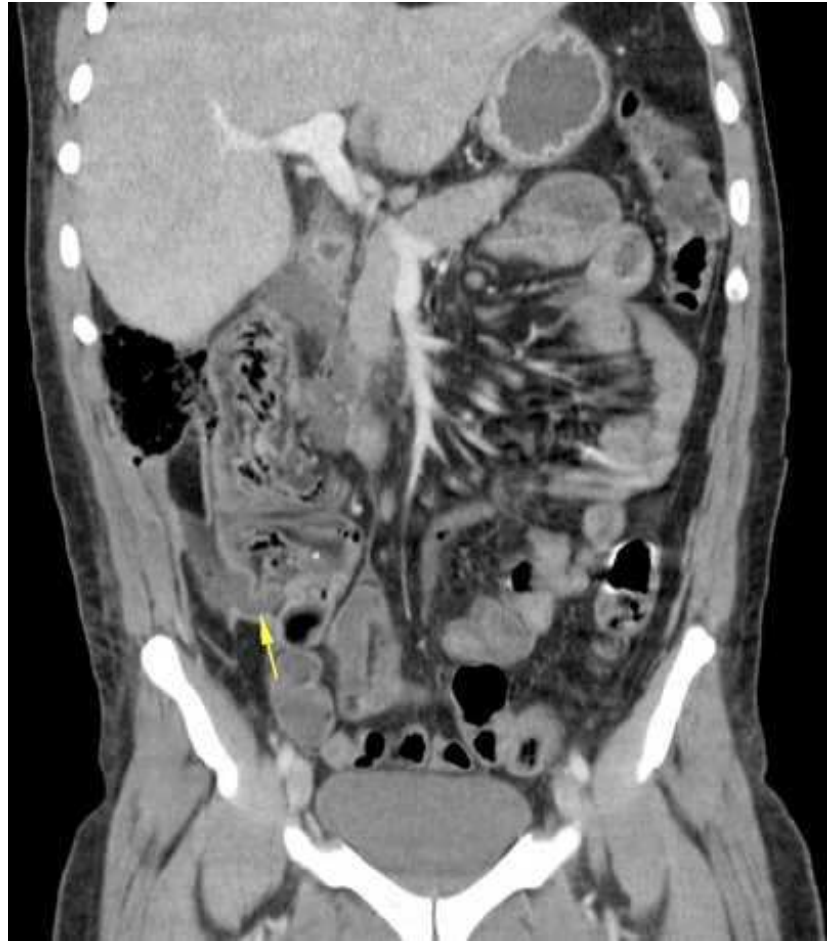

Figura 1: Tomografia computadorizada de abdome, plano coronal: $\mathrm{Na}$ região do coto apendicular, observa-se presença de líquido livre que se estende para o compartimento extra peritoneal.

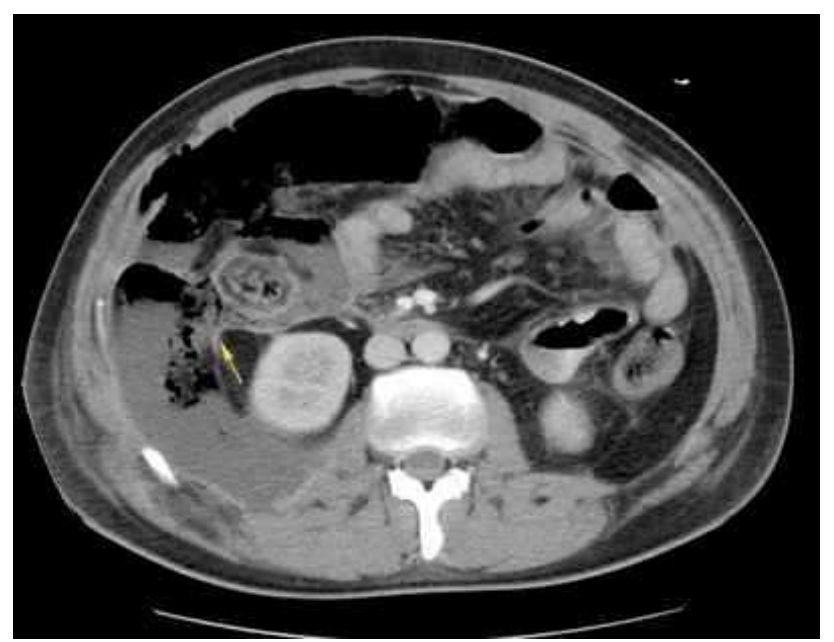

Figura 2: Tomografia computadorizada de abdome, corte axial: observa-se coleção hidro aérea circundando o ceco e o colo ascendente, com extensão para o compartimento extra peritoneal e subcutâneo adjacente.

Paciente foi submetido à laparotomia exploradora, sendo encontrada grande quantidade de secreção purulenta em cavidade abdominal, intensas aderências, perfuração em ceco na região de base apendicular, com necrose da região e dos planos adjacentes. Foi realizado desbridamento da região necrótica em ceco 
e sutura com pontos separados e, devido às condições da cavidade abdominal, optado por confecção de peritoniostomia a Barker. Já havia sido iniciada empiricamente antibioticoterapia antes da realização da primeira cirurgia, com ampicilina, gentamicina e metronidazol. Na cultura de secreção purulenta retirada da cavidade abdominal após primeira abordagem cirúrgica, identificou-se o crescimento de Escherichia coli resistente à ampicilina e ciprofloxacino, sendo feito o descalonamento dos antibióticos após o resultado.

Após primeira abordagem, o paciente necessitou de mais sete revisões da cavidade abdominal devido abscessos e fistulas intestinais, sendo que o sucesso no controle das mesmas foi realizado através do direcionamento das fistulas para linha média com sondas e curativos com pressão negativa. Devido a sepse abdominal de dificil controle, foi necessário manter antibioticoterapia de 14 dias de ampicilina e sulbactam por falha do primeiro tratamento. Em hemocultura posterior, cerca de 1 mês e meio após a primeira abordagem, foram identificados Cocos Gram positivos compativeis com Staphylococcus aureus (MRSA), e em cultura de secreção purulenta drenada de cavidade abdominal, identificado o crescimento de Escherichia coli ESBL. Foram então realizados 14 dias de teicoplanina e meropenem, com boa resposta clínica ao tratamento.

Após 130 dias de internamento hospitalar entre unidade de terapia intensiva e enfermaria, paciente teve alta hospitalar com hérnia abdominal planejada e fistulas entero-atmosféricas controladas com débito para bolsa de ostomia.

O relato teve a sua confecção autorizada pelo paciente através de termo de consentimento livre e esclarecido e foi devidamente submetido ao comitê de ética com aprovação - CAAE: 25603219.8.0000.0107.

\section{DISCUSSÃo}

A apendicite de coto é uma das raras complicações tardias após a apendicectomia, com incidência relatada de 1 em 50.000 casos $^{3}$. A literatura a respeito da apendicite de coto é escassa, e é baseada principalmente em relatos de caso e revisões sistemáticas. Assim como no caso relatado, essa literatura mostra que uma significativa porcentagem dos casos se enquadrou como difícil ou complicado, o que pode ser relacionado também a um atraso diagnóstico pela baixa suspeição, uma vez que se trata de um quadro muito semelhante a uma apendicite aguda comum, porém com uma apendicectomia prévia $^{4-6}$.

A apendicite de coto ocorre de meses até décadas após a apendicectomia ${ }^{3,4}$. Principalmente quando não identificada e tratada, a apendicite de coto pode evoluir com complicações graves como apendicite gangrenosa, flegmão, fístula cutânea, perfuração (principal complicação), abscesso intra-abdominal e sepse. A inclusão da apendicite de coto como diagnóstico diferencial da dor abdominal em QID (o sintoma mais comum e presente em 70 a $88 \%$ dos casos de apendicite de coto) é de grande importância, uma vez que devido ao seu baixo índice de suspeita o diagnóstico é muitas vezes retardado 5,6 .

Por ser mais sensivel e específica do que a ultrassonografia, excluir outras etiologias do abdome agudo e ser capaz de identificar achados inespecíficos como alterações inflamatórias pericecais, formação de abscesso, fluido na goteira paracólica direita, espessamento da parede cecal, massa ileocecal e, em alguns casos, como achado mais específico, a visualização do coto apendicular, a TC tem sido o método de preferência no diagnóstico de apendicite de coto ${ }^{5,7}$. Tal como acontece com a apendicite aguda, 24-36\% dos pacientes têm um estudo de imagem negativo ou inconclusivo, o que caracteriza a TC ainda como preferivel em casos ambíguos, pelo fato de fornecer mais detalhes ${ }^{6}$.

Há relatos de apendicite de coto após apendicectomia aberta com ligadura de coto, apendicectomia aberta com inversão de coto e também após apendicectomia laparoscópica na qual o coto apendicular é fechado com um endloop ou por grampeamento ${ }^{5}$. Apesar de menos preferida entre os cirurgiões, a técnica de sepultamento/inversão do coto apendicular teoricamente evitaria complicações tais quais a contaminação peritoneal, formação de aderências e a apendicite do coto. No entanto, não há evidências que apoiem a inversão versus ligadura simples, visto que as duas técnicas são equivalentes em 
relação ao tempo de duração da operação, morbimortalidade e tempo de internação pós-operatório6,8. Além disso, não existem evidências que apontem a via aberta ou laparoscópica como melhor técnica nesses casos. E embora a maioria dos casos de apendicite de coto ainda ocorra após apendicectomia por via aberta, ainda existe a ideia de que a apendicite de coto pode ser mais frequente com a abordagem laparoscópica, devido ao campo de visão bidimensional estreito e à ausência de feedback tátil15-7. Entretanto, não se pode afirmar que a apendicectomia por via aberta seja um fator de risco por levar a mais casos de apendicite de coto, uma vez que, como mencionado anteriormente a maioria das apendicectomias ainda é realizada por via laparotômica ${ }^{1}$.

Os principais fatores de risco para a apendicite de coto são a falha em identificar a base do apêndice durante a apendicectomia e deixar um coto do apêndice residual longo $(>0,5 \mathrm{~cm})^{6}$. Assim, para a prevenção da apendicite de coto, recomenda-se a ressecção do apêndice, deixando um coto $<3 \mathrm{~mm}$ de comprimento ${ }^{4,6}$. Isso pode ser feito através de uma boa identificação e visualização da junção apendicocecal, seguindo a convergência da tênia do ceco até a base do apêndice. Como alternativa para marcação da verdadeira base do apêndice, ainda pode-se dissecar e ligar o ramo recorrente da artéria apendicular ${ }^{3}$. Também é possivel alcançar uma boa visão da junção apendicocecal através da elevação do apêndice em direção à parede abdominal, proporcionando leve tensão, o que ajudará na dissecção dos planos de tecido significativamente inflamados ${ }^{7}$.

O tratamento de escolha para a apendicite de coto é hoje representado por uma segunda apendicectomia completa (apendicectomia com a ressecção completa do coto), e a maioria dos casos é tratada por apendicectomia total aberta ${ }^{5}$. Nos casos de apendicite de coto em que foi realizada TC com boa visualização da anatomia, incluindo a medida do comprimento do coto remanescente, recomenda-se a apendicectomia completa realizada laparoscopicamente guiada pelos achados da TC, em vez de pela via aberta ${ }^{7}$.

Embora venha sendo descrita já há mais de 70 anos, a apendicite de coto ainda é uma condição pouco conhecida e pouco lembrada pelos médicos 5 . Portanto, a apendicite de coto como hipótese diagnóstica em pacientes com apendicectomia prévia que apresentam suspeita de abdome agudo e sintoma de dor em FID não deve ser prontamente excluída, uma vez que, apesar de rara, pode levar a complicações graves.

\section{REFERÊNCIAS}

1. Santos FD, Cavasana GF, Campos TD. Perfil das apendicectomias realizadas no Sistema Público de Saúde do Brasil. Rev. Col. Bras. Cir. 2017;44(1):4-8. doi: 10.1590/010069912017001002 .

2. Dikicier E, Altintoprak F, Ozdemir K, Gundogdu K, Uzunoglu MY, Cakmak G, et al. Stump appendicitis: a retrospective review of 3130 consecutive appendectomy cases. World J Emerg Surg. 2018;13:22.

doi: 10.1186/s13017-018-0182-5.

3. Liang MK, Lo HG, Marks JL. Stump appendicitis: a comprehensive review of literature. Am Surg. 2006;72(2):162-6.

4. Ünlüer EE, Karagöz A, Sahin Y, Bilgin S, Oyar O. Diagnostic challenges with stump appendicitis. Am J Emerg Med. 2015;34(6):1188.e1-2.

doi: 10.1016/j.ajem.2015.11.037.

5. Geraci G, Lena A, D'Orazio B, et al. A rare clinical entity: stump appendicitis. Case report and complete review of literature. Clin Ter. 2019;170(6):e409e417. doi: 10.7417/CT.2019.2167.

6. Manatakis DK, Aheimastos V, Antonopoulou MI, et al. Unfinished Business: A Systematic Review of Stump Appendicitis. World J Surg. 2019;43(11):2756-61.

doi: $10.1007 / \mathrm{s} 00268-019-05101-z$.

7. Roberts KE, Starker LF, Duffy AJ, Bell RL, Bokhari J. Stump appendicitis: a surgeon's dilemma. JSLS. 2011;15(3):373-8. doi: $10.4293 / 108680811$ X131257333569 54.

8. Neves LJVA, Wainstein AJA, Mathias WC, Costa FPD, Castro JH, SavassiRocha PR. Ligadura simples ou ligadura com confecção de bolsa e sepultamento para tratamento do coto apendicular: estudo comparativo prospectivo randomizado. $\mathrm{ABCD}$, arq. 
bras. cir. dig. 2011;24(1):15-9. doi: 10.1590/S010267202011000100004.
Fonte de financiamento: Não

Conflito de interesses: Não

Data de Submissão: 22 Maio 2021

Decisão final: 11 Outubro 2021

Autor de Correspondência:

Juliana Sopchaki Fagundes

E-mail: jusopchaki@gmail.com 